\title{
To stand or not to stand? Implications of prolonged standing for perioperative nurses: A discussion paper
}

Follow this and additional works at: https://www.journal.acorn.org.au/jpn

Part of the Perioperative, Operating Room and Surgical Nursing Commons (c) (i)

This work is licensed under a Creative Commons Attribution 4.0 License.

\section{Recommended Citation}

Olynick, Katinka and Foran, Paula (2021) "To stand or not to stand? Implications of prolonged standing for perioperative nurses: A discussion paper," Journal of Perioperative Nursing: Vol. 34 : Iss. 4 , Article 8. Available at: https://doi.org/10.26550/2209-1092.1167

https://www.journal.acorn.org.au/jpn/vol34/iss4/8

This Discussion paper is brought to you for free and open access by Journal of Perioperative Nursing. It has been accepted for inclusion in Journal of Perioperative Nursing by an authorized editor of Journal of Perioperative Nursing. 


\section{Authors}

Katinka Olynick

RN, MACN

Dr Paula Foran

PhD, RN, FACORN, FACPAN, MACN

\title{
To stand or not to stand? Implications of prolonged standing for perioperative nurses: A discussion paper
}

\begin{abstract}
Perioperative nurses, including perianaesthesia, instrument and circulating nurses, stand for most of their shift - anywhere from one to eight hours at a time. This prolonged standing has been linked to negative effects on health, increasing the incidence of musculoskeletal disorders, such as lower back pain and neck pain, and cardiovascular diseases, such as oedema, varicose veins and venous pooling.
\end{abstract}

Given the impact these workplace injuries have on nurses, and on workplaces through financial costs associated with sick leave and/or workers compensation claims, surely prevention would be better than cure. While limited research exists to categorically suggest what prevention strategies are best, several options are available for consideration.

The use of anti-fatigue mats has been associated with lower incidence of back pain. It is suggested that perioperative nurses consider using sit-stand stools, if available, and compression socks at $15-20 \mathrm{mmHg}$, if standing in a static position for long periods.

Proper posture while standing - described as a neutral pelvis, natural thoracic curvature, flat abdomen, aligned shoulder, hip and ankles and an erect head - can assist in preventing disorders associated with prolonged standing. A combination of stretching and strength training for perioperative staff can help improve musculoskeletal symptoms experienced due to poor posture and tension, and the introduction of microbreaks has also seen improvements in concentration and comfort while reducing fatigue and discomfort.

This paper will discuss the health effects of prolonged standing and provide information about ergonomic interventions, compression socks or stockings, stretching programs and microbreaks for perioperative nursing teams to consider.

Key words: prolonged standing, anti-fatigue mat, perioperative nurse, health implications, musculoskeletal disorders, cardiovascular impairment, stretching program, microbreaks

\section{Introduction}

This paper will argue that current ergonomic aids and prevention strategies are essential to reduce the negative effects of prolonged standing experienced by perioperative nurses. Perioperative nurses, who are valued members of the perioperative team, expose their bodies to the risk of developing musculoskeletal disorders and soft tissue injuries due to their work environment? However, instrument and circulating nurses are at particular risk as they are required to stand for long periods of time and perform movements including lifting, pushing, pulling, twisting and retracting ${ }^{2}$. When carrying out 
their role, instrument nurses will maintain either a static posture or perform repetitive movements that are sustained for the duration of the surgery². To help prevent adverse effects on health, multiple ergonomic interventions have been studied to determine if they reduce these risks. Together with the health implications of prolonged standing, this paper will discuss ergonomic interventions, including anti-fatigue floor mats, sit-stand stools and postural training, as well as compression socks or stockings, stretching programs and microbreaks.

\section{Health implications}

During surgery, prolonged standing is a common occurrence that not only becomes uncomfortable over time but is also detrimental to health?. The combination of standing while twisting, lifting, retracting, pushing and pulling are classified as highrisk tasks for developing soft tissue and musculoskeletal injuries in the perioperative workplace ${ }^{2}$. Lower back pain was documented as being the most prevalent complaint of perioperative nurses, with over 60 per cent of perioperative nurses in a 2021 study suffering from this issue?.

The incidence of musculoskeletal injuries and discomfort - in particular, lower back pain - is a common health problem that is directly associated with prolonged standing, static positioning and repetitive movements 2,3 . The incidence of lower back pain in nurses is linked to increased sick leave and reduced productivity 4 . In a 2018 study that surveyed the prevalence of lower back pain in instrument and circulating nurses $(n=250)$, most respondents (84\%) reported at least one episode of lower back pain during the past year that was attributed to repetitive, static movements $(p<0.05)$ or assisting with patient positioning $(p<0.01)^{4}$.
Cardiovascular disease, chronic venous disorders and chronic vascular insufficiency are also health issues commonly associated with professions that require prolonged sitting and standing 5 . A 2020 study that documented symptoms of chronic venous insufficiency suffered by participants $(n=500)$ in careers requiring sitting or standing for more than four hours per day found that the most common complaint of health care workers $(n=111)$ was heaviness, swelling and numbness to the lower extremities ${ }^{5}$. Another study conducted in 2020 investigated the link between female nurses $(n=181)$ and prevalence of varicose veins ${ }^{6}$. A direct correlation was established between the standing time of nurses and the risk of developing varicose veins $(p=0.01)^{6}$. Furthermore, it was noted that for every hour of static standing the risk of developing varicose veins increased $(p=0.00)$, with 53.9 per cent of perioperative nurses in the study formally diagnosed with this condition. A Canadian study conducted over 12 years found that gender plays a role in incidence of chronic venous disorders, with women $(p<0.001)$ at a higher risk than men of developing cardiovascular disease due to prolonged standing $(p=0.03)^{7}$.

\section{Ergonomic interventions}

Throughout prolonged surgery the instrument and circulating nurses frequently stand unaided for the duration of the case and operating theatre floors, due to infection control requirements, are commonly rigid in nature ${ }^{8}$. Ergonomic interventions to reduce harmful effects of this rigidity include antifatigue mats, sit-stand stools and postural training.

Anti-fatigue mats are designed to provide a flexible surface that aims to disrupt stability and encourage muscle activation thus improving venous flow ${ }^{8}$. Aghazadeh et al. conducted a study that showed no significance in muscle activation when anti-fatigue mats were used, although there was a noted reduction in lower back pain $(p<0.05)^{3}$. Currently there are limited studies to conclude whether or not anti-fatigue mats are effective in improving musculoskeletal discomfort and injury9. There is also a lack of literature to suggest if age, gender and oral intake have a significant effect when using anti-fatigue mats? It is important to note that although anti-fatigue mats may provide pressure relief and increased comfort, they are also a hazard for tripping so caution should be taken when they are used during surgery ${ }^{9}$.

Sit-stand stools can improve comfort for the instrument or circulating nurse during surgery ${ }^{2}$. Using a sit-stand stool can reduce the weight load in nurses' legs, feet and back providing better body weight distribution, improving venous flow and reducing spinal loading 2,6 . A 2019 study ( $n=24)$ documented a hybrid position between sitting and standing as a favourable position for both genders $(p<0.005)$ in creating natural pelvic and lumbar angles and reducing body weight tension and loading ${ }^{10}$. While it is not always acceptable for the instrument or circulating nurse to use this equipment, a sit-stand stool should be used when appropriate ${ }^{10}$.

Prolonged standing with or without ergonomic aid can, over time, cause postural strain ${ }^{11}$ and poor posture is one of the leading causes of musculoskeletal pain in the operating theatre ${ }^{11}$. Proper posture while standing is when the pelvis is in a neutral position, the thoracic spine has its natural curves, the abdomen is flat, the shoulder, hip and ankles are aligned, and the head is erect ${ }^{11}$. A combination of stretching and 
strength training for perioperative staff can improve posture and aid in reducing musculoskeletal symptoms experienced due to poor posture ${ }^{11}$.

\section{Compression socks or stockings}

Compression socks are commonly used to assist in alleviating vascularrelated issues through increasing venous pressure, reducing oedema and improving blood flow ${ }^{12}$. There is a variety of compression socks and stockings available ranging from ankle and thigh to full stocking length, with multiple grades of compression ${ }^{13}$. A recent randomised trial conducted by Lee et al. showed that female nurses $(n=20)$ reported higher levels of satisfaction wearing thigh length compared to knee length compression socks $(p=0.041)^{13}$.

Belczak et al. studied the effects of $15-20 \mathrm{mmHG}$, and $20-30 \mathrm{mmHg}$ compression on individuals who work in roles requiring prolonged standing, sitting and a combination of the two ${ }^{13}$. The results showed that compression of 20-30 $\mathrm{mmHg}$ provided the biggest reduction in volumetric measurements for the sitting cohort $(p<0.001)^{14}$. For instrument and circulating nurses, compression of $15-20 \mathrm{mmHg}$ was recommended as this is more effective for improving venous flow and comfort when standing during surgery ${ }^{14,15}$. While compression socks and stockings have been found to be effective in relieving pain $(p=0.002)$ and aching $(p<0.0001)$ their tightness can make them difficult to don and cause discomfort with prolonged wear ${ }^{12,15}$.

\section{Stretching programs and microbreaks}

A study conducted in America found that although stretching can improve posture and relieve symptoms, there is a lack of education about and practice recommendations for postural awareness in the perioperative environment ${ }^{11}$.

An Australian study $(n=42)$ investigated the use of a stretching program involving multidisciplinary perioperative staff ${ }^{16}$. The program was based on previously successful stretching programs in other workplaces and was set up in a vacant recovery room bay, with orientation to the program being provided by a physiotherapist ${ }^{16}$. The study reported that before the stretching program was implemented a muskuloskeletal-related incident was four times more likely to happen in the perioperative department than in the rest of the hospital; after the stretching program was implemented this likelihood was reduced by 60 per cent (OR 0.4, 95\% Cl 0.1, 8.0 $p=0.01)^{16}$. More than 70 per cent of participants stated that the program was feasible and 85 per cent felt it was a good fit for the operating suite. However, a common problem reported by staff was that there was not enough time to access the program $^{16}$.

A group of surgeons combined stretching and microbreaks using evidence from other industries ${ }^{17}$. Findings revealed that microbreaks with exercise either provided no change or improvement in mental focus (88\%) and physical performance $(100 \%)$ of participants, with minimal disruption to the flow of surgical lists ${ }^{17}$. Taking microbreaks was documented as beneficial to the surgical team, allowing time to stretch and reduce fatigue and discomfort while not disrupting the flow of surgery or increasing surgical time ${ }^{17}$. Recommendations to include stretching and microbreaks into the operating room were praised by 87 per cent of surgeons $(n=56)^{17}$.

\section{Practice recommendations}

It is recommended that perioperative nurses, and the whole perioperative team, look closely at the range of strategies available to prevent the harmful effects of prolonged standing. Safe Work Australia identifies hazardous manual tasks that can lead to musculoskeletal disorders ${ }^{18}$. Many of the daily tasks carried out by perioperative nurses during surgery fall into the category of hazardous manual tasks as described by Safe Work Australia; however, currently there is no legislation in place requiring ergonomic aids to be used in health facilities. The Association of periOperative Registered Nurses (AORN) has published a set of guidelines that recommend nurses should not stand for more than 30 per cent of their shift, and proposes that pregnant nurses should spend no more than three hours in static positions, due to the risk of foetal harm². The introduction of Australian safety legislation would assist in compliance with using ergonomic interventions and measures to prevent cardiovascular disease associated with prolonged standing.

It is noted that while wearing compression socks or stockings is a simple intervention, using sit-stand stools is not always an option, as the instrument nurse is often required to stand while completing static and repetitive movements for lengthy surgeries ${ }^{2}$, and anti-fatigue mats may be tripping hazards.

Education about the importance of good posture and the introduction of exercise programs and microbreaks may also aid in the prevention of cardiovascular and musculoskeletal disorders. 


\section{Conclusion}

This discussion paper has argued that ergonomic interventions and prevention strategies are essential to reduce the harmful effects experienced by all perioperative nurses due to prolonged standing.

Currently, there is not enough research available to draw a definitive conclusion about the most appropriate method for reducing the effects on health that are associated with prolonged standing for the perioperative nurse. All ergonomic methods and strategies possess benefits that perioperative nurses can gain from when used correctly. A recommendation from this paper would be for more research to be conducted into this vitally important occupational health and safety concern.

Health facilities may ultimately be responsible for making ergonomic equipment available to employees and for introducing preventative stretching exercise programs and microbreaks. Given the impact of workplace injuries, on both nurses and their employers, prevention may be better and more cost effective than cure. It is time to look at prevention strategies in your workplace.

\section{Acknowledgment}

This paper was submitted to the University of Tasmania as part fulfilment of subject CNA803, Advanced Clinical Nursing Practice, for the Master of Clinical Nursing (Perioperative Nursing). The author sincerely wishes to thank Dr Paula Foran, unit coordinator, for her guidance throughout the course and work in preparing this paper for publication.

\section{References}

1. Clari M, Godono A, Garzaro G, Voglino G, Rosaru Gualano M et al. Prevalence of musculoskeletal disorders among perioperative nurses: A systematic review and META-analysis. BMC 2021;22(1):226.

2. Apple $B$, Letvak $S$. Ergonomic challenges in the perioperative setting. AORN J 2021;113(4): 339-348.

3. Aghazadeh J, Ghaderi M, Azghani MR, Khalkhali, HR, Allahyari T, Mohebbi I. Anti-fatigue mats, low back pain, and electromyography: An interventional study. IJOMEH 2015;28(2):347-356.

4. Jeyakumar AK, Segaran F. Prevalence and risk factors of low back pain and disability index among operating room nurses. JPN 2018;31(3):21-24.

5. Lastowiecka-Moras E. Standing and sitting postures at work and symptoms of venous insufficiency - results from questionnaires and a Doppler ultrasound study. JOSE 2020, DOI: $10.1080 / 10803548.2020 .1834232$.

6. Shakya R, Karmacharya RM, Shrestha $R$, Shrestha A. Varicose veins and the risk factors among nurses at Dhulikhel hospital: A cross sectional study. BMC Nurs ! 2020;19(8):1-7.

7. Smith P, Ma H, Glazier RH, Gilbert-Ouimet $M$, Mustard $C$. The relationship between occupational standing and sitting and incident heart disease over a 12-year period in Ontario, Canada. AM J Epidemiol 2018;187(1):27-33.

8. Speed G, Harris K, Keegal T. The effect of cushioning materials on musculoskeletal discomfort and fatigue during prolonged standing. Appl Ergon 2018;70:300-314.

9. Alaqeel M, Tanzer M. Improving ergonomics in the operating room for orthopaedic surgeons in order to reduce work-related musculoskeletal injuries. Ann Med Surg 2020;56(1):133-138.

10. Noguchi M, Glinka M, Mayberry GR, Noguchi K, Callaghan JP. Are hybrid sit-stand postures a good compromise between sitting and standing? Ergonomics 2019;62(6):811-822.

11. Winters JN, Sommer, NZ, Romanelli, MR, Marschik, C, Hulcher, L, Cutler BJ. Stretching and strength training to improve postural ergonomics and endurance in the operating room. PRS Global Open 2020;8(5)e2810.

12. Konschake $W$, Riebe $H$, Pediaditi P, Hasse $H$, Junger $M$, Lutze $S$. Compression in the treatment of chronic venous insufficiency: Efficacy depending on the length of the stocking. Clin Haemorheol Microcirc 2016;64(3):425-434.
13. Lee Y, Kim K, Kang S, Kim JY, Kim SG, Kim T et al. Compression stocking length effects on oedema, pain, and satisfaction in nursing students: A pilot randomized trial. Healthcare J 2020; 8(2):149.

14. Belczak CEQ, de Godoy JMP, Seidel SC, Ramos RN, Caffaro RA. Comparison of 15-20 $\mathrm{mmHg}$ versus 20-30 mmHg compression stockings in reducing occupational oedema in standing and seated healthy individuals. Open Access Journals 2018, issue 2018. Art. No.: 2053985. DOI: https://doi.org/10.1155/2018/2053985.

15. Rabe E, Partsch H, Hafner J, Lattimer C, Mosti G, Neumann M et al. Indications for medical compression stockings in venous and lymphatic disorders: An evidencebased consensus statement. Phlebology 2018;33(3):163-184.

16. King A, Campbell J, James C, Duff J. A workplace stretching program for the prevention of musculoskeletal disorders in perioperative staff: A mixed methods implementation study. JPN 2020; 33(4): e-3-e-10.

17. Hallbeck S, Lowndes BR, Bingener J, Abdelrahman AM, Yu D, Bartley A et al. The Impact of intraoperative microbreaks with exercise on surgeons: A multi-center cohort study. Appl Ergon 2017;60:334-341.

18. Safe Work Australia. Lifting, pushing and pulling (manual handling) [Internet]. Safe Work Australia: Canberra; 2020 [cited 2020 September 17]. Available from: https://www. safeworkaustralia.gov.au/manual-handling. 